\title{
Erratum
}

\section{Deep Level Study by Analysis of Thermal and Optical Transients in Semiconductor Junctions}

G. Vincent*

Laboratoire de Physique de la Matière ${ }^{\star \star}$,

Institut National des Sciences Appliquées de Lyon, 20, Avenue Albert Einstein,

F-69621 Villeurbanne Cédex, France

Appl. Phys. 23, 215-221 (1980)

Equation (12b) should read

$\frac{\Delta C}{C}=\frac{\gamma}{2} \frac{N_{T}}{n_{0}}\left(\Delta-\frac{\lambda}{l}\right)^{2}$.

In the caption of Fig. 2 the two relations, such as

$$
E_{F}^{*}-E_{T}=\beta^{2} V_{B} \quad \frac{e_{n}}{e_{n}+e_{p}} \frac{N_{T}}{n}=2 \alpha^{2},
$$

should be separated in each case.

The inequality in the 6 th line from top of the right-hand column of page 218 should read $\gamma N_{T} / n_{0} \ll 1$. 